\title{
The Role of Supportive Therapy in Pediatric Malignancies
}

\author{
Papp Zsuzsanna Erzsébet*, Horváth Mária-Adrienne \\ Mures County Hospital- Pediatric Department, University of Medicine and Pharmacy Tirgu Mureș, Romania
}

Childhood cancer is a major psycho-social and health problem. International study groups establish complex, efficient, and concrete Cytostatic Protocols for every cancer type. During chemotherapy patients become extremely vulnerable to infections, so it is necessary to complete the treatment with blood substitution, anti-infection medication, growth factors and other complementary products. Materials and Methods: We studied the importance of the wide palette of adjuvant therapy near the intensive cytostatic treatment in the period of March 2014-November 2015 at the hemato-oncology department in Pediatric Clinic of Mures County Hospital. Results: In this period we treated 20 children ( 9 female, 11 male) aged between 9 months-18 years. We had 15 cases of haemopathies (13 acute leukemia and two lymphomas), and five solid tumors. Packed red blood cells, platelets, and fresh frozen plasma were given in the aplastic period. A patient benefited, on average, a total of $70 \mathrm{ml} / \mathrm{kg}$ packed red blood cells and $50 \mathrm{U}$ platelets. For infection prophylaxis and treatment every child benefited associated infective medication. Discussions: Packed red blood cells, platelets, and fresh frozen plasma were given to patients with a deficiency in the ability to produce normal blood cells which are temporarily worsened by chemotherapy. Antibiotic and antifungal medications are given to all febrile and neutropenic patients. We use wide spectrum antibiotics in association for preventing sepsis. Growth factors are stimulating the bone marrow to increase leukocyte number. Since introducing additional immunostimulant medication, we observed a significant decrease of infection in the aplastic period. Conclusions: Oncology protocols use only 3-5 cytostatic drugs. Maintaining the patient's life during the treatment, it is necessary to use a large spectrum of supportive medications.

Keywords: child, cancer, supportive treatment

Received: 23 February 2016 / Accepted: 23 March 2016

\section{Introduction}

Despite all significant progress in diagnosis and treatment of pediatric malignant diseases, cancer remains a major psycho-social and health problem. The treatment protocols are complex, efficient and specific for every cancer type. During this period patients become extremely vulnerable to infections, so it is necessary to complete the treatment with additional products [1].

The average length of intensive chemotherapy is about 200 days. The complementary treatment consists of corticosteroids plus additional medications, antiemetics, antiinfectious treatments, blood product substitutions, intravenous immunoglobulins, human albumin, granulocyte colony stimulating factors, neuro roborant treatments, antihistaminics, psycho-supportive treatments [2].

Corticosteroids are a baseline in most protocols, for their antitumoral and antiallergic effect, and they help to maintain the leucocyte count. Undesirable side effects are gastritis, Cushing syndrome, hyperglycemia, vascular hypertension, osteoporosis, neuropathy, myopathy, edema, headache, psychosis, stretch marks, or other trophic disorders of the skin. Associated medication is needed such as gastroprotective (proton pump inhibitor+antacids), potassium, calcium, vitamin D3, low sodium+hypoglycemic diet, neuro roborants, and sedatives.

Neutropenic patients with/without fever need empiric antibiotic treatment. It is important to know that patients

* Correspondence to: Zsuzsanna Erzsébet Papp

E-mail: pappzsuzsi@freemail.hu who have neutropenia associated with corticosteroids do not show obvious clinical signs of infection. All patient who have fever receive antibiotic therapy, at a minimum, a third-generation cephalosporin. Other antibiotic agents are added if specific infections are documented [3]. Patients with persistent fever after 3-5 days of antibiotics should be given associated antifungal therapy. Most clinicians prescribe prophylactic antibiotics for neutropenic patients even if they are not febrile undergoing induction therapy.

Patients with malignancies have a deficiency in the ability to produce normal blood cells and they need substitution therapy. This deficiency is temporarily worsened by the addition of chemotherapy. If the $\mathrm{Hgb}$ value decreases under $8 \mathrm{~g} / \mathrm{dl}$, or the thrombocyte value is under $20 \times 10^{3 /}$ $\mu \mathrm{L}$, blood product administration is needed. In case of bleeding incidences or prolonged prothrombin time, fresh frozen plasma is also indicated.

Before administration of blood products, antihistaminic and hydrocortisone medication is recommended. All blood products must be irradiated to prevent viral infections and transfusion-related graft versus host disease.

Granulocyte colony stimulating factors (GCSF) are given to neutropenic patients to raise neutrophil counts, to shorten aplastic periods, and decrease nonviral infections prevalence without influence on late treatment response, remission period, or long term event free survival [4].

Intravenous immunoglobulin (IVIG) is indicated as replacement therapy in severe secondary immunodeficiencies combined with infections. Human albumin is given at low serum proteins for a better function of the immunity. 
Long-term corticosteroids associated with certain cytostatic medications such as Vincristine, have a proven neurotoxic effect, this justifies the neuro roborant medication.

Liver function is disrupted due to prolonged chemotherapy; hepatoprotective treatment improves transaminase values [5].

\section{Materials and methods}

By our retrospective descriptive study we observed the adjuvant treatments, substitution therapy by the necessary blood products, and anti-infective treatments per patient during the period March 2014-November 2015 in the oncology-hematology Department of Pediatric County Hospital in Targu Mures. Data was collected from the patients' files and correlated with clinical manifestations. Including criteria was age under 18 years and intensive cytostatic treatment for oncological illness; we excluded all the patients who were on maintenance therapy.

\section{Results}

In this period, we treated 20 children with intense chemotherapy ( 9 female, 11 male) aged between 9 months-18 years (mean: 7.18 years). Majority pathology was pre-B cell acute lymphoblastic leukemia- ALL (12 patients), followed by 3 cases of Wilms tumor- WT ( 1 case with of pulmonary lung metastasis), and 1 case of M2 acute myeloid

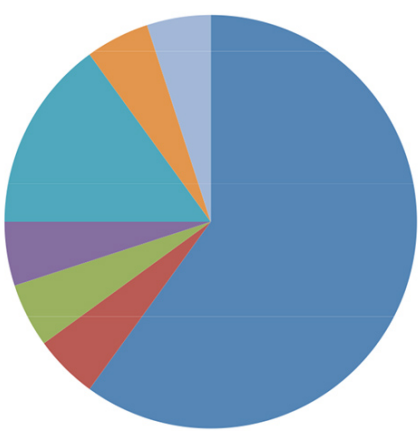

Acute lymfoblastic leukemia-12

Acute mieloblastic leukemia-1

Hodgkin lymphoma-1

Anaplastic large cell lymphoma-1

Wilms tumor-3

Desmoplastic small round cell tumor-1 leukemia- AML, Hodgkin lymphoma- HL, Anaplastic large cell Non Hodgkin lymphoma- ALCL, stage 4 desmoplastic small round cell tumor- DSRCT with liver metastasis, treated and relapsed presacral neuroblastoma-NB. (Figure 1) The sex ratio was equal. All of our patients were treated according to international Cytostatic Protocols.

In case of the malignant hemopathies, we found the following extreme blood parameters: $\mathrm{Hgb} 5 \mathrm{~g} / \mathrm{dL}$, total leucocyte count $0.02 \times 10^{3} / \mu \mathrm{L}$ and thrombocytes $2.5 \times 10^{3} / \mu \mathrm{L}$ (ALL). Solid tumor and lymphoma patients showed less severe aplastic periods.

Packed red blood cells, platelets, and fresh frozen plasma were given. A patient benefited, on average, a total of 70 $\mathrm{ml} / \mathrm{kg}$ packed red blood cells and $50 \mathrm{U}$ platelets (Figure. 2).

Antibiotics and antifungal medications were given to all neutropenic patients. We use wide spectrum antibiotic association to prevent sepsis. Every neutropenic episode, even if there were no signs of acute infection, needed at least a generation 2 cephalosporin, but concomitant with the increasing $\mathrm{C}$ reactive Protein values, associated with clinical signs of infections, we needed to complete with other, larger spectrum antibiotics. The mean value of the infections was 3 with the specification that the 12 year old high risk Philadelphia chromosome positive ALL patient and the 4.5 years old acute AML patient presented 7 bacterial infection episodes, mostly pneumonias, but also necrotizing enteritis. Other documented infections were panaritium, bacterial parotiditis and cutaneous infections at the insertion area of the central venous catheters. Figure 3 and 4 demonstrate that leukemic patients needed more antibiotic- antifungal medication associations than lymphoma and solid tumor patients in the aplastic period. In order to prevent Pneumocystis carinii infections, all of our patients were treated with trimethoprim/sulfamethoxazole 3/7 scheme, so none of them presented any signs of this parasite infection. Except one case of proven Pulmonary Aspergillosis by the 12 year old high risk ALL patient (clinical signs as cough, hypoxemia, pinkish saliva/sputum associated with positive culture from bronchial secretion), which caused his death in one week, we had no other fungal infections. Despite use of

Fig. 1. Pathology distribution

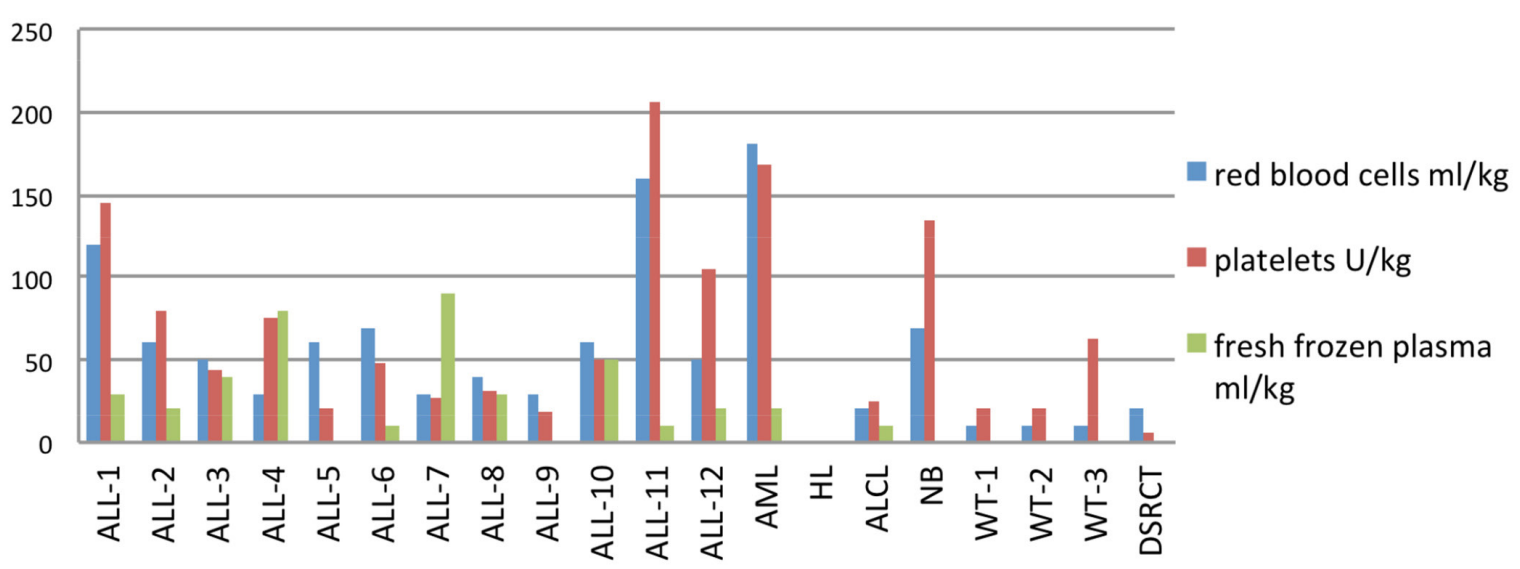

Fig. 2. Blood products necessity 


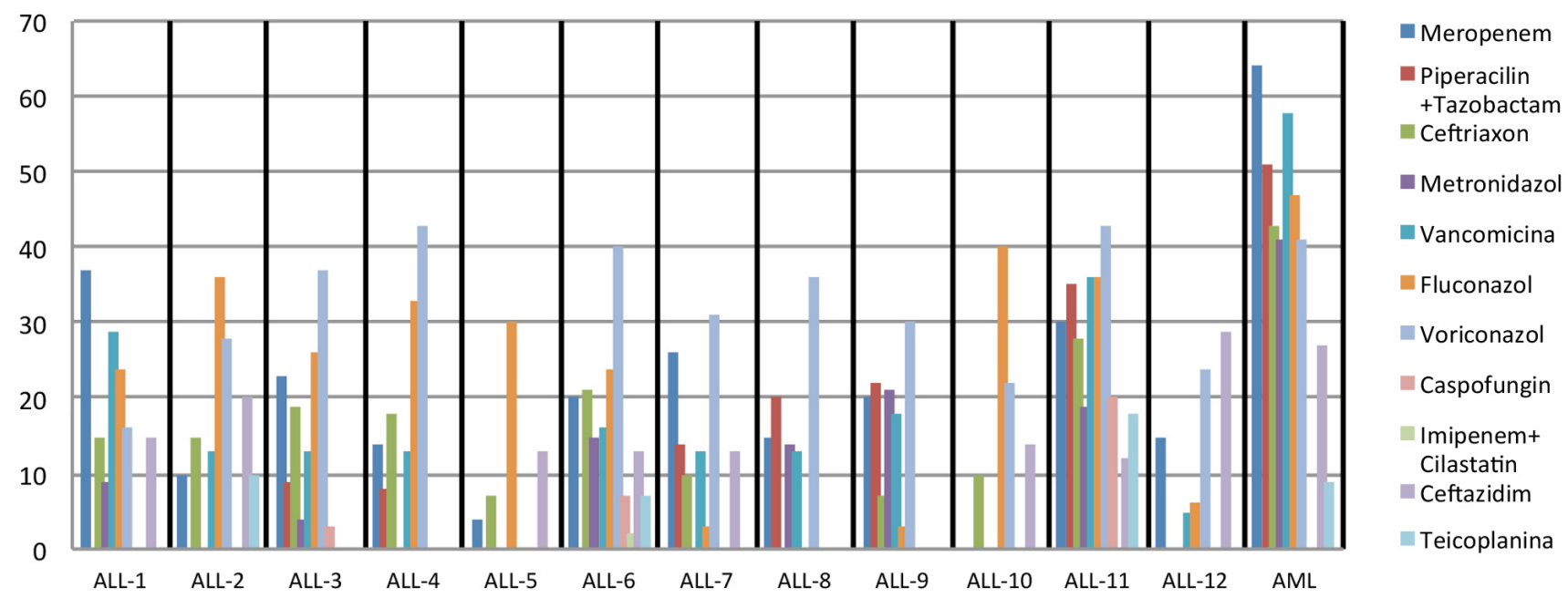

Fig. 3. Antiinfectious treatment- acute leukemias

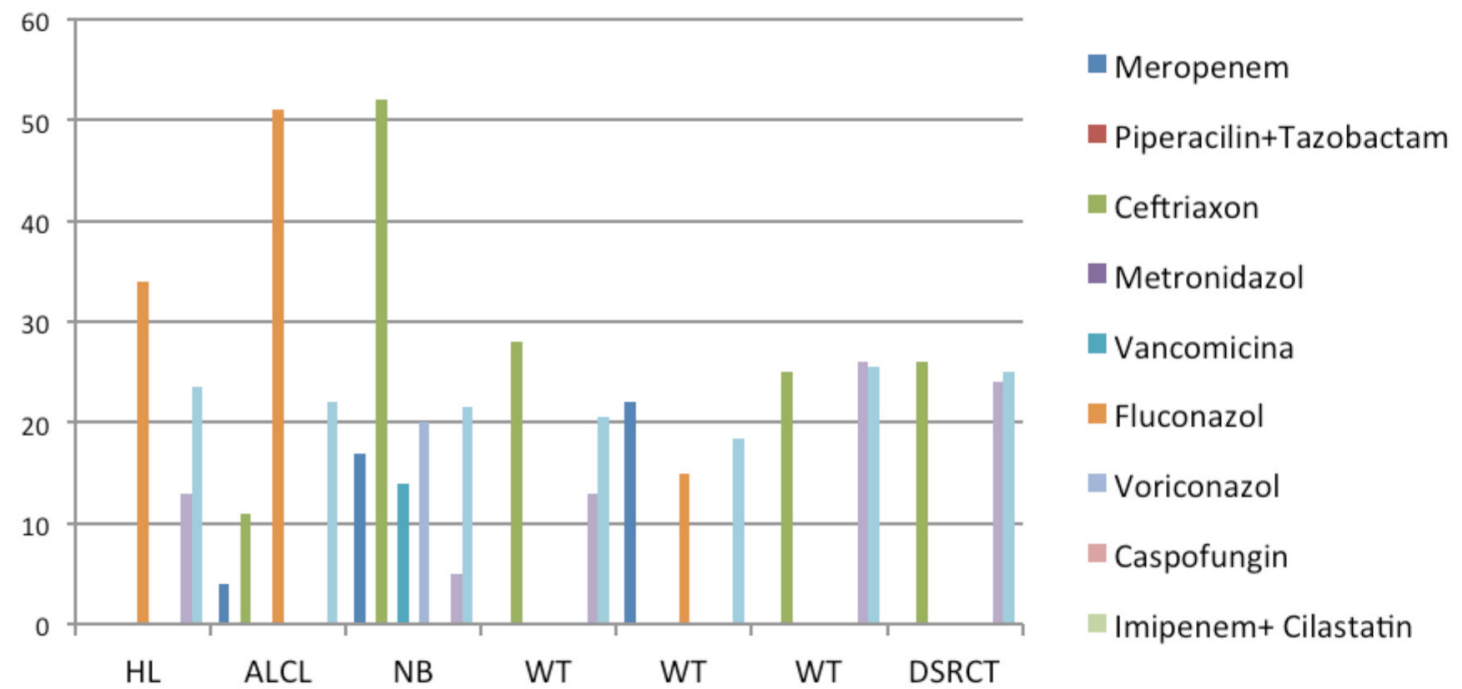

Fig. 4. Antiinfectious treatment - lymphomas and solid tumors

intravenous Voriconazol and Caspofungin administration, the patient could not be saved.

Regarding the viral infections we had only one case of varicella virus reactivation- zona zoster at the 16 years old Hodgkin Lymphoma patient, who after the diagnosis and introducing $80 \mathrm{mg} / \mathrm{kg} /$ day acyclovir treatment was released. The rest of the patients who were contacts were treated also with the same dose of acyclovir for preventing the development of clinical signs of viral infections.

With the exception of the two lymphoma patients (Hodgkin and non-Hodgkin), all children needed intravenous immunoglobulin substitution. Two patients - a 4.5 years old girl with AML and a 12 years old boy with high-risk Philadelphia chromosome positive ALL- received seven doses of $2.5 \mathrm{~g}$ iv immunoglobulin. If total plasma protein values decreased under $50 \mathrm{~g} / \mathrm{l}$, we administered human albumin products (Figure 5). Associated hepatoprotective treatment was necessary for all patients because of the increased transaminase levels (Figure 6). Extreme high values were found in the 18 years old young male with stage IV abdominal desmoplastic small round cell tumor DSRCT with liver metastasis. The most frequently used hepatoprotective drug, in our department, was Ursodeoxycholic acid (Figure 7).

In order to prevent another important side effect of chemotherapy, every patient received a correct dose of 0.15 $\mathrm{mg} / \mathrm{kg}$ iv ondansetron 30 minutes before cytostatics.

All patients benefited additional supportive treatments with po. antioxidant medication, p.o. colloidal silver solutions, po. and iv vitamins and po. extract of Whitania Somnifera, Trapa natans, Vitis vinifera, Pyrus Malus.

Psycho-supportive treatment is offered by a professional organization, which has been collaborating with our department, for one year.

\section{Discussions}

In our study, the pathology distribution data respected the literature that says that ALL is most common childhood malignancy followed by central nervous system tumors and lymphomas [6]

Cytopenias were corrected with packed red cells, platelets, and subcutaneous GCSF. The substitution therapy indications and the techniques were conform transfusion protocols. We observed a higher necessity of blood prod- 


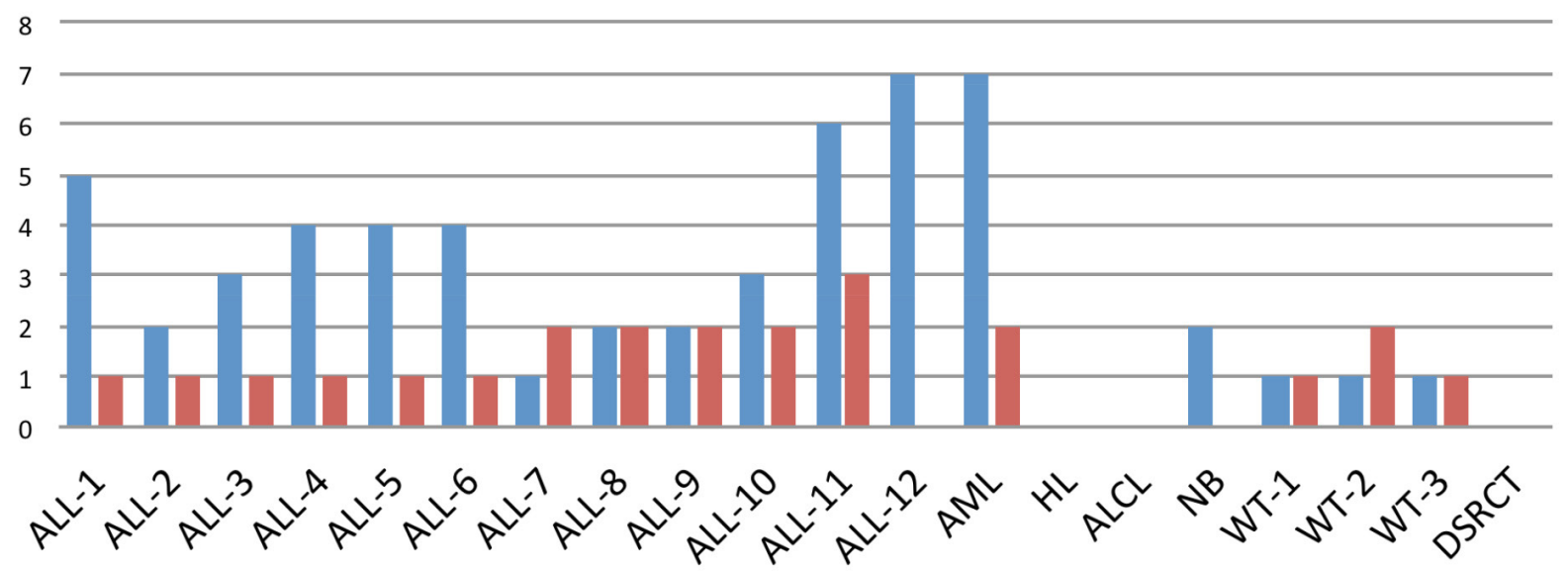

ivig $2.5 \mathrm{~g}$ human albumin $20 \% 0.5 \mathrm{~g} / \mathrm{kg}$

Fig. 5. Ivig and human albumin administration

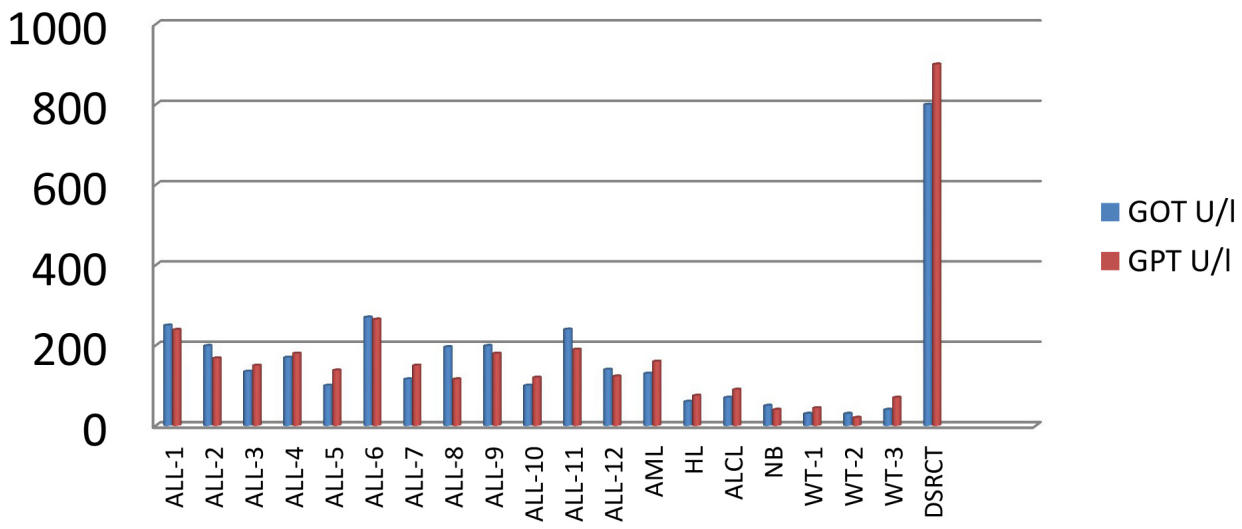

Fig. 6. Extreme transaminaze values

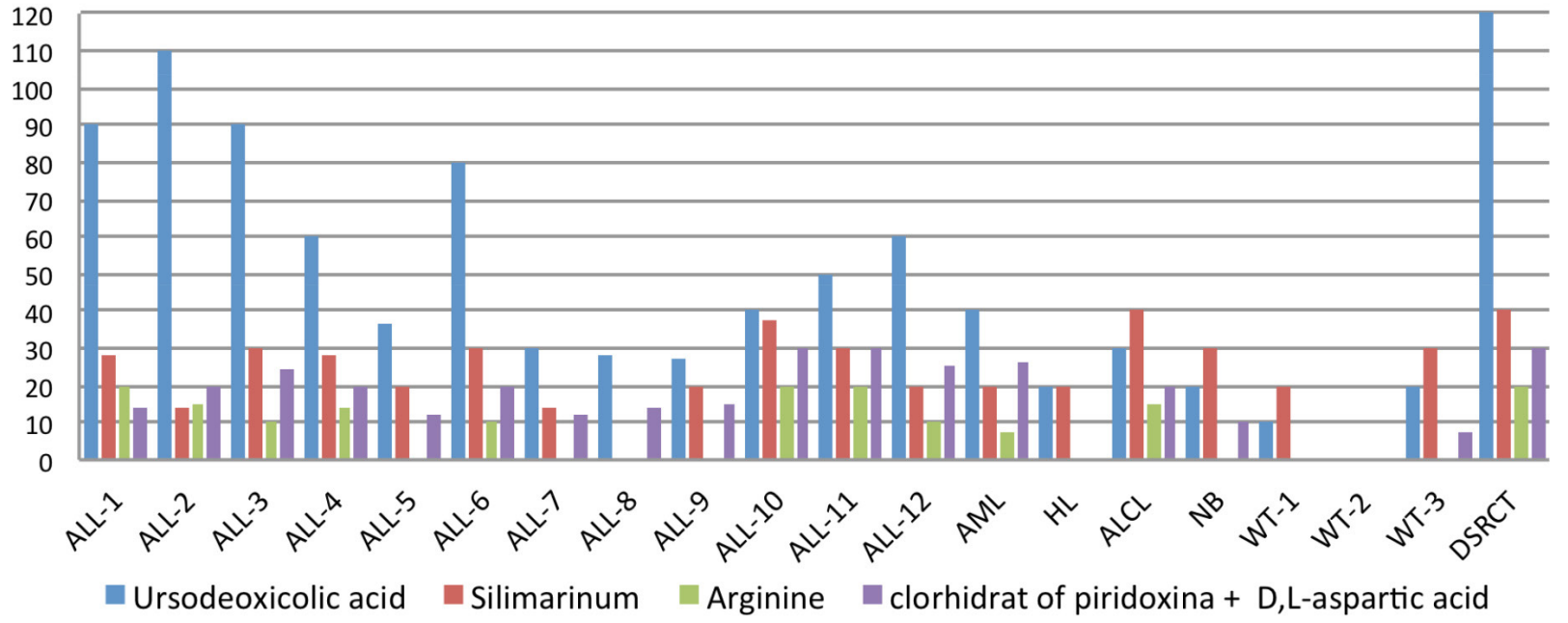

Fig. 7. Hepatoprotective treatment

ucts and growth hormone in patients with hematological malignancies than in patients with lymphomas and solid tumors, this is explained by the fact that in leukemias the bone marrow is invaded by the tumoral blast cells, so there is no space remained for the healthy cell lines, and in solid tumors the bone marrow is intact, so the 3 main blood cell lineages growth, differentiation and maturation is undisturbed $[7,8]$. Before blood product administration antiallergic medication was needed, as cetirizinum, loratadinum and intravenous corticotherapy. If a cancer patient before the moment of precise diagnosis is with certain cytopenia and needs blood products substitution, we need to avoid corticotherapy administration till the bone marrow is analyzed, because of the temporary antitumoral effect of it. During blood product administration whenever can anaphylactic shock appear. In our study we observed 11 allergic episode associated with platelet administration (7 ALL patients, 4 of them repeated the allergic incidence). The 
symptoms were hyperthermia, flushing, shiver, headache at 5-10 minutes after beginning the platelet transfusion. Discontinuing the transfusion, administration of $5-8 \mathrm{mg} /$ $\mathrm{kg} /$ dosis iv Hidrocortisone, $10 \mathrm{mg} / \mathrm{kg} /$ dosis iv paracetamol and saline perfusion settled the symptoms and we never had anaphylactic shock developed.

Bacterial infections in the immunocompromised, neutropenic subject occurre very easy. Treatment consists of associated antibiotics (CG2-3, Meropenem, anti-staphylococcus treatment ) and immune support by intravenous immunoglobulin.(9) The longer the aplastic period is, the more IVIG is needed, so in our study the most severe aplastic periods were by the AML and high risk ALL patient observed.

Aspergillus can affect immunocompromised patients and can cause different infections with different localisations. Pulmonary Aspergillosis (cough, low-grade fever / high fever, dyspnea, hemoptysis) is difficult to treat with a severe prognosis despite associated antifungal medication. Our case of proven Pulmonary Aspergillosis by the 12 year old high risk ALL patient benefitted 5 months of continuous fluconazole/voriconazol prevention scheme, but we had a poor compliance from the child and the mother, so at the moment of the pulmonary infection he was without antifungal medication. The clinical signs were fulminant as cough, hypoxemia, pinkish saliva/sputum, we initiated voriconazole+caspofungin intravenous treatment, but after a week we lost him. Data from literature also recommends fungal infection prophylaxis, it is used amphotericin B as first line defense, but this medication is not available in Romania, so we choose fluconazole/voriconazole $[10,11]$.

To prevent Pneumocystis carinii pneumonia all patients receive prophylaxis with trimethoprim/ sulfamethoxazole three years, three days / week (weekend days). None of our patients included in the study presented any signs of this pulmonary infection. Classical symptoms are fever, nonproductive cough, and progressive shortness of breath. Chest radiograph demonstrates bilateral, symmetric reticular (interstitial) or granular opacities. Evident laboratory findings are not available if the suspicion rises and the moment when the symptoms appear is already late, so it is easier to administrate a primary prophylaxis with this cheap sulfonamide than to treat afterwards with expensive combined antibiotics [12].

Viral infections, specially varicella can cause lethal meningitis at an immunocompromised organism. All patients who are contacts or even develop symptoms of viral infectious diseases are isolated and acyclovir therapy is instituted $80 \mathrm{mg} / \mathrm{kg} /$ day for one month. It is recommended, that in case of no varicella infection in the case/ family history the sibling should be vaccinated for varicella in order to prevent the infection in the family of the oncologic patient [13]

Antiemetic medication is essential for an oncologic patient, there can be no chemotherapy administration without a correct dose of antiemetics, so every patient benefited of ondansetron before cytostatics. There was one case of a
12 years old girl diagnosed with Wilms tumor who despite of administering 30 minutes before cytostatics intravenous ondansetron, she presented repeated vomiting, even if she only saw the infusion kit, we considered her reaction having a mental/psychological component too.

Hepatotoxicity is common by the multidrug treatment benefitting patient, because of the liver metabolisation of the most pharmaceuticals. In our study the highest values were found in DSRCT patient because of the chemotherapy and the secondary liver determinations.

Since introducing immunostimulating additional medication, we observed a significant decrease of infection in the aplastic period [14]

\section{Conclusions}

The oncologic patient is extremely vulnerable. Intensive chemotherapy causes secondary bone marrow aplasia, which requires blood substitution, anti-infective therapy and GCSF. Treatment Protocols are using 3-5 cytotoxic drugs in a cure, but to keep the patient alive during and between cytostatic periods, it is necessary to use a wide range of supportive medication.

It is important to know that an onco-pediatric patient has to ingest every day an average of 8-10 different medication, fact which is not considered polypharmacy, it is an absolute necessity.

\section{Abrevations}

ALCL- anaplastic large cell lymphoma

ALL - acute lymfoblastic leukemia

AML- acute mieloblastic leukemia

DSRCT- desmoplastic small round cell tumor

FFP- fresh frozen plasma

GCSF- granulocyte colony stimulating factor

IVIG- intravenous immunoglobulin

HL- Hodgkin lymphoma

NB- Neuroblastoma

NHL- Non Hodgkin Lymphoma

po- per oral

RBC- red blood cells

WT- Wilms tumor

\section{Acknowledgments}

We wish to send our special thanks to the University of Medicine and Pharmacy Tg Mures and our Clinic staff, both nurses and physician colleagues .

\section{Conflicts of interest}

The authors report no conflicts of interest.

\section{References}

1. Marinca M, Miron L, Miron I. Terapia suportiva si simptomatica, in L. Miron, Terapia oncologica-optiuni bazate pe evidente. Institutul European, lasi, 2008,799-862

2. Segal BH, Walsh J, Gea-Bannaloche JC, et al. Infections in the cancer patients, in Devita VT Jr, Rosenberg S, Hellman SA, eds. Cancer: Principles and practice of oncology. 7th ed Philadelphia: Lippincott, Williams\&Wilkins, 2005,2461-2514 
3. Safdar N, Crnich CJ, Maki DG. Infectious complications of cancer therapy, in Chang AE, Ganz PA, Hayes DF, eds. Oncology- an evidence based approach. New York, Springer, 2006,1363-1400.

4. Richard A. Larson, Richard K. Dodge, Charles A. Linker et al. A Randomized Controlled Trial of Filgrastim During Remission Induction and Consolidation Chemotherapy for Adults With Acute Lymphoblastic Leukemia: CALGB Study 9111. Blood Journal 1998;92(5):1556-1564

5. Anca Bacârea, Bogdana Dorcioman, Minodora Dobreanu et al. Tratamentul leucemiei acute mieloide - între beneficiu terapeutic si patologie iatrogenă - prezentare de caz. Revista Română de Medicină de Laborator Martie 2008;10(1):33-39

6. Primic-Zakelj M. Cancer epidemiology, in Scrijvers D, Senn HJ, Mellstedt $\mathrm{H}$, Zakotnk B ( eds) European Society of Medical Oncology Handbook of Cancer Prevention. Informa Healthcare 2008,1-28.

7. Ph.Lanzkowsky. Manual of pediatric hematology and oncology-fourth edition, 2005;548- 560

8. Kondo M. Lymphoid and myeloid lineage commitment in multipotent hematopoietic progenitors. Immunological reviews. 2010;238(1):37-46. doi:10.1111/j.1600-065X.2010.00963

9. Caselli D, Paolicchi O. Empiric antibiotic therapy in a child with cancer and suspected septicemia. Pediatric Reports. 2012;4(1):e2. doi:10.4081/ pr.2012.e2.

10. Garcia JB, Lei X, Wierda W, et al. Pneumonia during Remission Induction Chemotherapy in Patients with Acute Leukemia. Annals of the American Thoracic Society. 2013;10(5):432-440. doi:10.1513/AnnalsATS.2013040970C

11. Barkati S, Dufresne SF, Bélanger S, et al. Incidence of invasive aspergillosis following remission-induction chemotherapy for acute leukemia: a retrospective cohort study in a single Canadian tertiary care centre. CMAJ Open. 2014;2(2):E86-E93. doi:10.9778/cmajo.20130062.

12. Huang L. Clinical and translational research in Pneumocystis and Pneumocystis pneumonia. Parasite : journal de la Société Française de Parasitologie. 2011;18(1):3-11. doi:10.1051/parasite/2011181003

13. Forbes HJ, Bhaskaran K, Thomas SL, et al. Quantification of risk factors for herpes zoster: population based case-control study. BMJ : British Medical Journal. 2014;348:g2911. doi:10.1136/bmj.g2911.

14. Hernández-García S, Effect of Oncoxin Oral Solution in HER2Overexpressing Breast Cancer. Nutr Cancer. 2015;67(7):1159-69. doi: 10.1080/01635581.2015.1068819. Epub 2015 Aug 4. 